\title{
STRUCTURE OF BIFURCATED SOLUTIONS OF TWO-DIMENSIONAL INFINITE PRANDTL NUMBER CONVECTION WITH NO-SLIP BOUNDARY CONDITIONS
}

\author{
JUNGHO PARK
}

\begin{abstract}
We consider the two-dimensional infinite Prandtl number convection problem with no-slip boundary conditions. The existence of a bifurcation from the trivial solution to an attractor $\Sigma_{R}$ was proved by Park [15]. The no-stress case has been examined in [16]. We prove in this paper that the bifurcated attractor $\Sigma_{R}$ consists of only one cycle of steady state solutions and it is homeomorphic to $S^{1}$. By thoroughly investigating the structure and transitions the solutions of the infinite Prandtl number convection problem in physical space, we confirm that the bifurcated solutions are indeed structurally stable. We show what the asymptotic structure of the bifurcated solutions looks like. This bifurcation analysis is based on a new notion of bifurcation, called attractor bifurcation, and structural stability is derived using geometric theories of incompressible flows.
\end{abstract}

\section{INTRODUCTION}

In this paper we explore the structure of bifurcated solutions of the Bénard convection with no-slip boundary conditions which mean that the fluid flow does not move on the top and bottom boundaries. The same problem has been studied under no-stress boundary conditions [16] and much of the work in this paper is similar to the no-stress case. However, no-slip conditions require new ideas because no-slip boundary conditions do not allow for an explicit solution of the linearized eigenvalue problem.

Rayleigh-Bénard convection, that is, a buoyancy-driven convection in a fluid layer heated from below and cooled from above, is one of the prime examples of bifurcating high-dimensional systems. It has long been a subject of intense theoretical and experimental study and has been applied to many different areas of study such as meteorology, geophysics, and astrophysics.

1991 Mathematics Subject Classification. 35Q, 35B, 37L, 76E.

Key words and phrases. Rayleigh-Bénard Convection, bifurcation, structure of solutions, structural stability, infinite Prandtl number, no-slip boundary conditions.

The work was supported by NYIT Institutional Support of Research and Creativity Grants. 
The governing equations are the following Boussinesq equations:

$$
\begin{aligned}
& \frac{\partial u}{\partial t}+(u \cdot \nabla) u+\rho_{0}^{-1} \nabla p-\nu \Delta u=-g k\left[1-\alpha\left(T-\bar{T}_{0}\right)\right], \\
& \frac{\partial T}{\partial t}+(u \cdot \nabla) T-\kappa \Delta T=0 \\
& \nabla \cdot u=0
\end{aligned}
$$

where $\nu$ is the kinematic viscosity, $\kappa$ is the thermal diffusivity, $\alpha$ is the coefficient of thermal expansion of the fluid, $g$ is the acceleration due to gravity, $u=\left(u_{1}, u_{2}\right)$ is the velocity field, $p$ is the pressure function, $T$ is the temperature field and $k=(0,1)$ is the unit vector in $x_{2}$-direction.

To make the equations non-dimensional, let

$$
\begin{aligned}
x & =h x^{\prime}, \\
t & =h^{2} t^{\prime} / \kappa, \\
u & =\kappa u^{\prime} / h, \\
T & =\beta h\left(T^{\prime} / \sqrt{R}\right)+\bar{T}_{0}-\beta h x_{2}^{\prime}, \\
p & =\rho_{0} k^{2} p^{\prime} / h^{2}+p_{0}-g \rho_{0}\left(h x_{2}^{\prime}+\alpha \beta h^{2}\left(x_{2}^{\prime}\right)^{2} / 2\right), \\
P_{r} & =\nu / \kappa .
\end{aligned}
$$

Omitting the primes, the equations (1.1)-(1.3) can be rewritten as follows:

$$
\begin{aligned}
& \frac{1}{P_{r}}\left[\frac{\partial u}{\partial t}+(u \cdot \nabla) u\right]+\nabla p-\Delta u-R T k=0, \\
& \frac{\partial T}{\partial t}+(u \cdot \nabla) T-\Delta T=0 \\
& \nabla \cdot u=0 .
\end{aligned}
$$

In the Boussinesq equations, we have two important numbers: the Rayleigh number,

$$
R=\frac{g \alpha\left(T_{2}-T_{1}\right) h^{3}}{\nu \kappa}
$$

which measures the ratio of overall buoyancy force to the damping coefficients, and the Prandtl number,

$$
P_{r}=\frac{\nu}{\kappa}
$$

which measures the relative importance of kinematic viscosity over thermal diffusivity. Here, $h$ is the distance between two plates confining the fluid and $T_{2}-T_{1}$ is the temperature difference between the bottom and top plates.

Due to the fact that mathematical information is very limited, the complicated equations have been simplified. For example, the infinite Prandtl number limit of the Boussinesq equations has been used as the standard model for the convection of earth's mantle, where it is argued that $P_{r}$ could be of the order $10^{24}$, as well as for many gasses under high pressure. A Broader rationale for investigating the infinite Prandtl number convection 
is based on the observation of both the linear and weakly nonlinear theories; that fluids with $P_{r}>1$ convect in a similar fashion. Moreover, the infinite Prandtl number model of convection can also be justified as the limit of the Boussinesq approximation to the Rayleigh-Bénard convection as the Prandtl number approaches infinity $[23,24]$.

In the limit of the infinite Prandtl number, the inertial terms in the momentum equation can be dropped, thus we are left with a linear dependence of the velocity field on temperature:

$$
\begin{aligned}
& \nabla p-\Delta u-R T k=0, \\
& \frac{\partial T}{\partial t}+(u \cdot \nabla) T-\Delta T=0, \\
& \nabla \cdot u=0 .
\end{aligned}
$$

The basic linear profiles of (1.7)-(1.9) are steady state solutions given by

$$
\begin{aligned}
& u=0, \\
& T=1-x_{2}, \\
& p=p_{0}+R\left(x_{2}-\frac{x_{2}^{2}}{2}\right) k .
\end{aligned}
$$

Let $q$ be the difference between $p$ and the steady state solutions, and let $\theta$ be the difference between $T$ and the steady states solutions, i.e, $p=$ $p_{0}+R\left(x_{2}-\frac{x_{2}^{2}}{2}\right) k+q$ and $T=\left(1-x_{2}\right)+\theta$. Then the equations for the perturbation of these trivial solutions are derived as

$$
\begin{aligned}
& \nabla q-\Delta u-R \theta k=0, \\
& \frac{\partial \theta}{\partial t}+(u \cdot \nabla) \theta-\Delta \theta-u_{2}=0, \\
& \nabla \cdot u=0 .
\end{aligned}
$$

Let $T=\sqrt{R} \theta$ and $p=q$. Then we have

$$
\begin{aligned}
& \nabla p-\Delta u-\sqrt{R} T k=0, \\
& \frac{\partial T}{\partial t}+(u \cdot \nabla) T-\Delta T-\sqrt{R} u_{2}=0, \\
& \nabla \cdot u=0 .
\end{aligned}
$$

Since the velocity field in (1.10) is linearly dependent on the temperature field in the infinite Prandtl number convection, the velocity field has a behavior that is much more regular than the finite Prandtl number model. Thus this is a distinct advantage of studying this model. In particular, by investigating the structure of the attractor generated only by $T$, it is possible to reconstruct the structure of the attractor in terms of $(u, T)$, which has the same topological structure as is obtained in terms of only $T$. 
We impose the periodic boundary condition with spatial periods $L$ in the horizontal direction $x_{1}$ :

$$
(u, T)(x, t)=(u, T)\left(x_{1}+k L, x_{2}, t\right), \text { for any } k \in \mathbb{Z} .
$$

The top and bottom plates are assumed to be no-slip(Dirichlet):

$$
u=0, \quad T=0 \quad \text { at } \quad x_{2}=0,1 .
$$

For an initial value problem we also provide an initial value as

$$
\left.(u, T)\right|_{t=0}=\left(u_{0}, T_{0}\right) .
$$

From a physical point of view, the two-dimensional convection problem can be considered an idealized model for many physical phenomena, including the Walker circulation over the tropics [19], which has the same topological structure as the cells given in Figure 4.1. Simulations of the RayleighBénard convection with the infinite Prandtl number and high Rayleigh numbers in spherical shell geometry have often been carried out to understand the thermal structure of the mantle and the evolution of the Earth [25]. They also tell us that the convection cells have almost uniform size for the lower Rayleigh number, but become irregular for the higher Rayleigh number. Branstator-Kushnir waves of atmospheric dynamics $[1,10]$ have similar topological structure as given in Figure 4.2.

Extensive mathematical studies have been conducted for the RayleighBénard convection since Rayleigh's work. In particular, for the RayleighBénard convection with the finite Prandtl numbers, readers are referred to Chandrasekhar [2], to Drazin and Reid [5] for linear theories; to Foias, Manley and Temam [7] for the existence and physical bounds of attractors; to Rabinowitz [18] for the existence of rectangular solutions; and to Ma and Wang [12] for attractor bifurcation. For the case regarding the infinite Prandtl number, the readers are referred to Constantin and Doering $[3,4]$ for the upper bounds of the minimal conduction value; to Schnaubelt and Busse [21] for two-dimensional convection rolls; to Keken [9] and to Yanagisawa and Tamagishi [25] for mantle and spherical shell convection studies, respectively; to X. Wang [23, 24] for the justification of the infinite Prandtl number convection as the infinite Prandtl number limit of the Boussinesq equations; and to Park [15, 16] for the attractor bifurcation theory of the infinite Prandtl number convection.

T. Ma and S. Wang [12] recently developed a new notion of bifurcation theory called attractor bifurcation. The existence of bifurcation from the trivial solution to an attractor $\Sigma_{R}$ of the infinite Prandtl number convection was achieved by the author [15] using the attractor bifurcation theories. In this paper, the following two problems will be addressed:

(1) the classification of the structure of $\Sigma_{R}$,

(2) the transition of the bifurcated solutions in physical space.

For the first result, we use an approximation formula for the center manifold function given in [12]. Of course, the key ingredient of the analysis is 
to derive the reduction equation for our problem. For the second result, it is essential to study the topological structure of the divergence-free vector fields for 2D-incompressible flows governed by the Navier-Stokes equations or Euler equations. Such a result would involve a specific connection between solutions of the equations and flow structure in physical space, i.e., this area of research links the kinematics to the dynamics of fluid flows.

As a result, we have a bifurcation from the trivial state to an attractor $\Sigma_{R}$ as $R$ crosses the critical Rayleigh number $R_{c}$, the first eigenvalue of the eigenvalue problem of linearized equations [15]. Next, we prove that $\Sigma_{R}$ is homeomorphic to $S^{1}$ and that $\Sigma_{R}$ consists of steady state solutions for $R_{c}<R<R_{c}+\varepsilon$ for some $\varepsilon>0$. These results can be obtained by analyzing eigenvectors in terms of the temperature field $T$ and later on, the velocity field $u(T)$ associated with the temperature $T$ can be reconstructed by the eigenvectors of $T$. Thanks to the geometric theory of $2 \mathrm{D}$ incompressible flows [13], the structure and its transitions between the convection states in physical space is analyzed. Through this, we prove that the associated velocity field is structurally stable and show what the asymptotic structure looks like. This leads us, in particular, to a rigorous justification of the structure as the cells given in Figure 4.1 and the structure as the zonally moving meandering flows in Figure 4.2. The main results of this paper are the followings.

Theorem 1.1. For the problem (1.10)-(1.12) with (1.13)-(1.15), we have the following assertions: for $R>R_{c}$, the equation bifurcates from the trivial solution $(T, R)=\left(0, R_{c}\right)$ to an attractor $\Sigma_{R}$ which is homeomorphic to $S^{1}$. $\Sigma_{R}$ consists of exactly one cycle of steady state solutions whose structure in physical space is shown as in Figure 4.1.

Theorem 1.2. For any $\left(u_{0}\left(T_{0}\right), T_{0}\right) \in \widetilde{H} \times H \backslash(\Gamma \cup E)$, there exists a time $t_{0} \geq 0$ such that for any $t \geq t_{0}$, the associated vector field $u\left(t, \Psi_{0}\right)$ is structurally stable and is topologically equivalent to the structure as shown in Figure 4.2, where $\Psi=\left(u\left(t, \Psi_{0}\right), T\left(t, \Psi_{0}\right)\right)$ is the solution of (1.10)-(1.14) with initial data $\Psi_{0}=\left(u_{0}\left(T_{0}\right), T_{0}\right)$ and $\Gamma$ is the stable manifold of $(u(T), T)=0$ with co-dimension 2 in $\widetilde{H} \times H$ and

$$
\begin{gathered}
\widetilde{H}=\left\{u \in L^{2}(\Omega)^{2} \mid \nabla \cdot u=0, u_{1} \text { is periodic in } x_{1}\right. \text { direction } \\
\text { and } \left.u_{2}=0 \text { at } x_{2}=0,1\right\}, \\
E=\left\{(u, T) \in \widetilde{H} \times H \mid \int_{0}^{1} u_{1} d x_{2}=0\right\} .
\end{gathered}
$$

The above two theorems will be proved in Section 3 and Section 4, respectively.

It is worthwhile to mention that the above results can only be obtained by the attractor bifurcation theories. The classical bifurcation theory including symmetry arguments implies that the bifurcated attractor contains a circle of steady states. We need, however, the new bifurcated theory to prove in particular that the bifurcated attractors are exactly of type $S^{1}$. 
The paper is organized as follows. After introducing the infinite Prandtl number convection and its mathematical settings in Section 2, we describe the center manifold approximation and bifurcation theories for the infinite Prandtl number convection in Section 3, followed by a topological structure of the bifurcated solutions. Then we study the structural stability and the asymptotic structure of the bifurcated solutions in Section 4.

\section{Bénard Problem and its Mathematical Setting}

In this paper we deal with the Boussinesq equations on the non-dimensional domain $\Omega=\mathbb{R} \times(0,1) \subset \mathbb{R}^{2}$. Since (1.10) together with (1.12) is a Stokes equation, we have the solution $u=u(T)$ under the above boundary condition. Replacing $u$ and its second component $u_{2}$ in (1.11) by the solution which depends on $T$, we obtain the following equation

$$
\frac{\partial T}{\partial t}+(u(T) \cdot \nabla) T-\Delta T-\sqrt{R} u_{2}(T)=0,
$$

where $u(T)$ and $u_{2}(T)$ satisfy (1.10).

Adjusting boundary and initial conditions yields

$$
\begin{gathered}
\left\{\begin{array}{l}
T=0 \quad \text { at } x_{2}=0,1, \\
T(x, t)=T\left(x_{1}+k L, x_{2}, t\right), \text { for } x \in \Omega,
\end{array}\right. \\
\left.T\right|_{t=0}=T_{0} .
\end{gathered}
$$

Instead of considering the bifurcation problem (1.10)-(1.12) with (1.13)(1.15), we consider only (2.1) with (2.2) and (2.3), which depends only on $T$. The velocity field $u(T)$ associated with $T$ will be reconstructed later on.

We shall recall here the functional setting of the equation (2.1) with the boundary and initial conditions (2.2) and (2.3). Let

$$
H=L^{2}(\Omega) \quad \text { and } \quad H_{1}=H_{0}^{1}(\Omega) \cap H^{2}(\Omega),
$$

where $H_{0}^{1}(\Omega)$ is the space of functions in $H^{1}(\Omega)$, which vanish at $x_{2}=0,1$ and are periodic in $x_{1}$-direction.

Thanks to the existence result, we can define a semi-group

$$
S(t): T_{0} \rightarrow T(t),
$$

which enjoys the semi-group properties.

In the bifurcation theory for the infinite Prandtl number convection, the critical Rayleigh number, denoted by $R_{c}$, plays a key role. We consider the linearized equations of (2.1) with the boundary conditions (2.2),

$$
-\Delta T-\sqrt{R} u_{2}(T)=0,
$$

where $u_{2}(T)$ satisfies (1.10).

Since this eigenvalue problem for the Rayleigh number $R$ is symmetric, all eigenvalues $R_{k}$ with multiplicities $m_{k}$ of (2.4) with (2.2) are real numbers, and

$$
0<R_{1}<\cdots<R_{k}<R_{k+1}<\cdots
$$


in the sense that

$$
\left\{\begin{array}{l}
-\Delta u+\nabla p-\lambda T k=0 \\
-\Delta T-\lambda u_{2}=0 \\
\nabla \cdot u=0
\end{array}\right.
$$

is equivalent to

$$
\int_{\Omega}[\nabla u \cdot \nabla \widetilde{u}+\nabla T \cdot \nabla \widetilde{T}] d x=\lambda \int_{\Omega}\left[T \widetilde{u}_{2}+u_{2} \widetilde{T}\right] d x
$$

where $\lambda=\sqrt{R}$.

The first eigenvalue $R_{1}$ depends on periods $L$. It is known that there is a period $L_{c}$ which minimizes $R_{1}(L)$. We denote the critical Rayleigh number by $R_{c}$. Let the multiplicity of $R_{c}$ be $m_{1}=m(m \geq 1)$ and the first eigenvectors $T_{1}, \cdots, T_{m}$ of (2.4) be orthonormal, such that

$$
\left\langle T_{i}, T_{j}\right\rangle_{H}=\int_{\Omega} T_{i} \cdot T_{j} d x=\delta_{i j} .
$$

Then $E_{0}$, the first eigenspace of (2.4) with (2.2) is

$$
E_{0}=\left\{\sum_{k=1}^{m} \alpha_{k} T_{k} \mid \alpha_{k} \in \mathbb{R}, 1 \leq k \leq m\right\} .
$$

Let $H$ and $H_{1}$ be defined as above. Define $L_{\lambda}=-A+B_{\lambda}: H_{1} \rightarrow H$ and $G: H_{1} \rightarrow H$ by

$$
\left\{\begin{array}{l}
A(T)=-\Delta T \\
B_{\lambda}(T)=\lambda u_{2}(T) \\
G(T)=-(u(T) \cdot \nabla) T
\end{array}\right.
$$

where $\lambda=\sqrt{R}$. Then we have an operator equation, which is equivalent to the Boussinesq equation (2.1):

$$
\frac{d T}{d t}=L_{\lambda} T+G(T)
$$

3. Center Manifold and Attractor Bifurcation Theory

3.1. Center manifold approximation. The purpose of this section is to recall some results of the center manifold theory under general settings, i.e., general function spaces and general functional equations. It is a powerful tool for the reduction method and for the dynamic bifurcation of abstract nonlinear evolution equations developed in [12].

Let $H$ and $H_{1}$ be two arbitrary Hilbert spaces, and $H_{1} \hookrightarrow H$ be a dense and compact inclusion. Consider the following nonlinear evolution equations

$$
\begin{aligned}
& \frac{d v}{d t}=L_{\lambda} v+G(v, \lambda), \\
& v(0)=v_{0},
\end{aligned}
$$


where $v: H \times[0, \infty) \rightarrow H$ is the unknown function, $\lambda \in \mathbb{R}$ is the system parameter, and $L_{\lambda}: H_{1} \rightarrow H$ are the parameterized linear completely continuous fields continuously depending on $\lambda \in \mathbb{R}$, which satisfy

$$
\left\{\begin{array}{l}
L_{\lambda}=-A+B_{\lambda} \text { a sectorial operator, } \\
A: H_{1} \rightarrow H \text { a linear homeomorphism, } \\
B_{\lambda}: H_{1} \rightarrow H \text { the parameterized linear compact operators. }
\end{array}\right.
$$

We can see that $L_{\lambda}$ generates an analytic semi-group $\left\{e^{-t L_{\lambda}}\right\}_{t \geq 0}$ and then we can define fractional power operators $L_{\lambda}^{\alpha}$ for any $0 \leq \alpha \leq 1$ with the domain $H_{\alpha}=D\left(L_{\lambda}^{\alpha}\right)$ such that $H_{\alpha_{1}} \subset H_{\alpha_{2}}$ if $\alpha_{2}<\alpha_{1}$, and $H_{0}=H$.

We now assume that the nonlinear terms $G(\cdot, \lambda): H_{\alpha} \rightarrow H$ for some $0 \leq \alpha<1$ are a family of parameterized $C^{r}$ bounded operators $(r \geq 1)$ continuously depending on the parameter $\lambda \in \mathbb{R}$, such that

$$
G(v, \lambda)=o\left(\|v\|_{H_{\alpha}}\right), \quad \forall \lambda \in \mathbb{R} .
$$

For the linear operator $A$, we assume that there exists a real eigenvalue sequence $\left\{\rho_{k}\right\} \subset \mathbb{R}$ and an eigenvector sequence $\left\{e_{k}\right\} \subset H_{1}$ such that

$$
\left\{\begin{array}{l}
A e_{k}=\rho_{k} e_{k}, \\
0<\rho_{1} \leq \rho_{2} \leq \cdots, \\
\rho_{k} \rightarrow \infty(k \rightarrow \infty),
\end{array}\right.
$$

where $\left\{e_{k}\right\}$ is an orthogonal basis of $H$.

For the compact operator $B_{\lambda}: H_{1} \rightarrow H$, we also assume that there is a constant $0<\theta<1$ such that

$$
B_{\lambda}: H_{\theta} \longrightarrow H \text { bounded, } \forall \lambda \in \mathbb{R} .
$$

We know that the operator $L=-A+B_{\lambda}$ satisfying (3.5) and (3.6) is a sectorial operator. It generates an analytic semigroup $\left\{S_{\lambda}(t)\right\}_{t \geq 0}$. Then the solution of (3.1) and (3.2) can be expressed as

$$
v\left(t, v_{0}\right)=S_{\lambda}(t) v_{0}, \quad t \geq 0 .
$$

We assume that the spaces $H_{1}$ and $H$ can be decomposed into

$$
\left\{\begin{array}{l}
H_{1}=E_{1}^{\lambda} \oplus E_{2}^{\lambda}, \quad \operatorname{dim} E_{1}^{\lambda}<\infty, \quad \text { near } \lambda_{0} \in \mathbb{R}^{1}, \\
H=\widetilde{E}_{1}^{\lambda} \oplus \widetilde{E}_{2}^{\lambda}, \quad \widetilde{E}_{1}^{\lambda}=E_{1}^{\lambda}, \quad \widetilde{E}_{2}^{\lambda}=\text { closure of } E_{2}^{\lambda} \text { in } H,
\end{array}\right.
$$

where $E_{1}^{\lambda}$ and $E_{2}^{\lambda}$ are two invariant subspaces of $L_{\lambda}$, i.e., $L_{\lambda}$ can be decomposed into $L_{\lambda}=\mathcal{L}_{1}^{\lambda} \oplus \mathcal{L}_{2}^{\lambda}$ such that for any $\lambda$ near $\lambda_{0}$,

$$
\left\{\begin{array}{l}
\mathcal{L}_{1}^{\lambda}=\left.L_{\lambda}\right|_{E_{1}^{\lambda}}: E_{1}^{\lambda} \rightarrow \widetilde{E}_{1}^{\lambda}, \\
\mathcal{L}_{2}^{\lambda}=\left.L_{\lambda}\right|_{E_{2}^{\lambda}}: E_{2}^{\lambda} \rightarrow \widetilde{E}_{2}^{\lambda},
\end{array}\right.
$$

where all eigenvalues of $\mathcal{L}_{2}^{\lambda}$ possess negative real parts, and all eigenvalues of $\mathcal{L}_{1}^{\lambda}$ possess nonnegative real parts at $\lambda=\lambda_{0}$. 
Thus, for $\lambda$ near $\lambda_{0},(3.1)$ can be rewritten as

$$
\left\{\begin{array}{l}
\frac{d x}{d t}=\mathcal{L}_{1}^{\lambda} x+G_{1}(x, y, \lambda), \\
\frac{d y}{d t}=\mathcal{L}_{2}^{\lambda} y+G_{2}(x, y, \lambda),
\end{array}\right.
$$

where $v=x+y \in H_{1}, x \in E_{1}^{\lambda}, y \in E_{2}^{\lambda}, G_{i}(x, y, \lambda)=P_{i} G(v, \lambda)$, and $P_{i}: H \rightarrow \widetilde{E}_{i}$ are canonical projections. Furthermore, we let

$$
E_{2}^{\lambda}(\alpha)=E_{2}^{\lambda} \cap H_{\alpha},
$$

with $\alpha$ given by (3.4).

Theorem 3.1. (Center Manifold Theorem) Assume (3.4)-(3.8). Then there exists a neighborhood of $\lambda_{0}$ given by $\left|\lambda-\lambda_{0}\right|\langle\delta$ for some $\delta>0$, a neighborhood $B_{\lambda} \subset E_{1}^{\lambda}$ of $x=0$, and a $C^{1}$ function $\Phi(\cdot, \lambda): B_{\lambda} \rightarrow E_{2}^{\lambda}(\alpha)$, depending continuously on $\lambda$, such that

(1) $\Phi(0, \lambda)=0, D_{x} \Phi(0, \lambda)=0$.

(2) The set

$$
M_{\lambda}=\left\{(x, y) \in H_{1} \mid x \in B_{\lambda}, y=\Phi(x, \lambda) \in E_{2}^{\lambda}(\alpha)\right\},
$$

called the center manifold, is locally invariant for (3.1), i.e., for any $v_{0} \in M_{\lambda}$,

$$
v_{\lambda}\left(t, v_{0}\right) \in M_{\lambda}, \quad \forall 0 \leq t<t_{v_{0}}
$$

for some $t_{v_{0}}>0$, where $v_{\lambda}\left(t, v_{0}\right)$ is the solution of (3.1).

(3) If $\left(x_{\lambda}(t), y_{\lambda}(t)\right)$ is a solution of (3.9), then there are $\beta_{\lambda}>0$ and $k_{\lambda}>0$ with $k_{\lambda}$ depending on $\left(x_{\lambda}(0), y_{\lambda}(0)\right)$, such that

$$
\left\|y_{\lambda}(t)-\Phi\left(x_{\lambda}(t), \lambda\right)\right\|_{H} \leq k_{\lambda} e^{-\beta_{\lambda} t} .
$$

By the help of the Center Manifold Theorem, we obtain the following bifurcation equation reduced to the finite dimensional system

$$
\frac{d x}{d t}=\mathcal{L}_{1}^{\lambda} x+G_{1}(x+\Phi(x), \lambda)
$$

for $x \in B_{\lambda} \subset E_{1}^{\lambda}$.

Now we recall an approximation of the center manifold function which will be used in the proof of Theorem 1.1; see [12] for details. Let the nonlinear operator $G$ be given by

$$
G(v, \lambda)=G_{k}(v, \lambda)+o\left(|v|^{k}\right)
$$

where $G_{k}(v, \lambda)$ is a $k$-multilinear operator $(k \geq 2)$.

Theorem 3.2. [12] Under the conditions in Theorem 3.1 and (3.10), we have the following center manifold function approximation:

$$
\Phi(v, \lambda)=\left(-\mathcal{L}_{2}^{\lambda}\right)^{-1} P_{2} G_{k}(v, \lambda)+O\left(|\operatorname{Re} \beta(\lambda)| \cdot\|v\|^{k}\right)+o\left(\|v\|^{k}\right),
$$

where $\beta(\lambda)=\left(\beta_{1}(\lambda), \cdots, \beta_{m}(\lambda)\right)$ are the eigenvalues of $\mathcal{L}_{1}^{\lambda}$. 
Consider the case where $L_{\lambda}: H_{1} \rightarrow H$ is symmetric. Then the eigenvalues are real, and the eigenvectors form an orthogonal basis of $H$. Therefore, we have

$$
\begin{aligned}
& v=x+y \in E_{1}^{\lambda} \oplus E_{2}^{\lambda}, \\
& x=\sum_{i=1}^{m} x_{i} e_{i} \in E_{1}^{\lambda}, \\
& y=\sum_{i=m+1}^{\infty} x_{i} e_{i} \in E_{2}^{\lambda} .
\end{aligned}
$$

Then near $\lambda=\lambda_{0}$, the formula in Theorem 3.2, can be expressed as follows:

$$
\Phi(x, \lambda)=\sum_{j=m+1}^{\infty} \Phi_{j}(x, \lambda) e_{j}+O\left(|\operatorname{Re} \beta(\lambda)| \cdot\|x\|^{k}\right)+o\left(\|x\|^{k}\right),
$$

where

$$
\begin{aligned}
\Phi_{j}(x, \lambda) & =-\frac{1}{\beta_{j}(\lambda)} \sum_{1 \leq j_{1}, \cdots, j_{k} \leq m} a_{j_{1} \cdots j_{k}}^{j} x_{j_{1}} \cdots x_{j_{k}}, \\
a_{j_{1} \cdots j_{k}}^{j} & =<G_{k}\left(e_{j_{1}}, \cdots, e_{j_{k}}, \lambda\right), e_{j}>_{H} .
\end{aligned}
$$

\subsection{Attractor bifurcation theory for the infinite Prandtl number} convection. In this subsection, we shall recall the attractor bifurcation theory of the infinite Prandtl number convection [15] and provide a sufficient condition which implies that the bifurcated attractor of the system (3.1) is homeomorphic to $S^{1}$.

Definition 3.3. A set $\Sigma \subset H$ is called an invariant set of (3.1) if $S(t) \Sigma=\Sigma$ for any $t \geq 0$. An invariant set $\Sigma \subset H$ of (3.1) is said to be an attractor if $\Sigma$ is compact and there exists a neighborhood $U \subset H$ of $\Sigma$ such that for any $\varphi \in U$ we have

$$
\lim _{t \rightarrow \infty} \operatorname{dist}_{H}(v(t, \varphi), \Sigma)=0 .
$$

The largest open set $U$ satisfying (3.12) is called the basin of attraction of $\Sigma$.

Definition 3.4. (1) We say that the equation (3.1) bifurcates from $(v, \lambda)=$ $\left(0, \lambda_{0}\right)$ to an invariant set $\Omega_{\lambda}$, if there exists a sequence of invariant sets $\left\{\Omega_{\lambda_{n}}\right\}$ of (3.1), $0 \notin \Omega_{\lambda_{n}}$, such that

$$
\begin{gathered}
\lim _{n \rightarrow \infty} \lambda_{n}=\lambda_{0}, \\
\lim _{n \rightarrow \infty} \max _{x \in \Omega_{\lambda_{n}}}|x|=0 .
\end{gathered}
$$

(2) If the invariant set $\Omega_{\lambda}$ is an attractor of (3.1), then the bifurcation is called attractor bifurcation.

(3) If $\Omega_{\lambda}$ is an attractor and is homotopy equivalent to an $m$-dimensional sphere $S^{m}$, then the bifurcation is called $S^{m}$-attractor bifurcation. 
Let the eigenvalues (counting multiplicity) of $L_{\lambda}$ be given by

$$
\beta_{k}(\lambda) \in \mathbb{C} \quad(k \geq 1) .
$$

Suppose that

$$
\begin{gathered}
\operatorname{Re} \beta_{i}(\lambda)\left\{\begin{array}{ll}
<0 & \text { if } \lambda<\lambda_{0}, \\
=0 & \text { if } \lambda=\lambda_{0} \\
>0 & \text { if } \lambda>\lambda_{0},
\end{array} \quad(1 \leq i \leq m),\right. \\
\operatorname{Re} \beta_{j}\left(\lambda_{0}\right)<0 \quad(m+1 \leq j) .
\end{gathered}
$$

Let the eigenspace of $L_{\lambda}$ at $\lambda_{0}$ be

$$
E_{0}=\bigcup_{i=1}^{m}\left\{v \in H_{1} \mid\left(L_{\lambda_{0}}-\beta_{i}\left(\lambda_{0}\right)\right)^{k} v=0, k=1,2, \cdots\right\} .
$$

The following dynamic bifurcation theorem for (2.1) was proved based on the principle of exchange of stabilities and asymptotic stability of trivial solution [15].

Theorem 3.5. [15] For the problem (2.1) with (2.2), we have the following assertions:

(1) If $R \leq R_{c}$, the steady state $T=0$ is a globally asymptotically stable equilibrium point of the equation.

(2) The equation (2.1) bifurcates from $(T, R)=\left(0, R_{c}\right)$ to an attractor $\Sigma_{R}$ for $R>R_{c}$, with $m-1 \leq \operatorname{dim} \Sigma_{R} \leq m$, which is connected when $m>0$.

(3) For any $T \in \Sigma_{R}$, the associated velocity field $u=u(T)$, which is achieved from a given $T$, can be expressed as

$$
u=\sum_{k=1}^{m} \alpha_{k} e_{k}+o\left(\sum_{k=1}^{m} \alpha_{k} e_{k}\right)
$$

where $e_{k}$ are eigenvectors of (2.1) corresponding to each $T_{k}$.

(4) The attractor $\Sigma_{R}$ has the homotopy type of an $(m-1)$-dimensional sphere $S^{m-1}$ provided $\Sigma_{R}$ is a finite simplicial complex.

(5) For any bounded open set $U \subset L^{2}(\Omega)$ with $0 \in U$ there is an $\varepsilon>0$ such that as $R_{c}<R<R_{c}+\varepsilon$, the attractor $\Sigma_{R}$ attracts $U / \Gamma$ in $L^{2}(\Omega)$, where $\Gamma$ is the stable manifold of $T=0$ with co-dimension $m$.

Remark 3.6. We can easily see that Theorem 3.5 is true for the 2D-Bénard problem as well as for the 3D-Bénard problem. It is also true under any combination of two boundary conditions, no-slip and no-stress, on top or bottom plates.

So far, we have introduced some crucial definitions and theorems which have established the existence of bifurcation. We now introduce another theorem related to the structure of the bifurcated solutions. 
Let $v$ be a two-dimensional $C^{r}(r \geq 1)$ vector field given by

$$
v_{\lambda}(x)=\lambda x-G(x, \lambda),
$$

for $x \in \mathbb{R}^{2}$. Here, $G(x, \lambda)$ is defined as in (3.10) and satisfies an inequality

$$
C_{1}|x|^{k+1} \leq<G_{k}(x, \lambda), x>_{H} \leq C_{2}|x|^{k+1},
$$

for some constants $0<C_{1}<C_{2}$ and $k=2 m+1, m \geq 1$.

Theorem 3.7. [12] Under the condition (3.17), the vector field (3.16) bifurcates from $(x, \lambda)=\left(0, \lambda_{0}\right)$ to an attractor $\Sigma_{\lambda}$ for $\lambda>\lambda_{0}$, which is homeomorphic to $S^{1}$. Moreover, one and only one of the following is true.

(1) $\Sigma_{\lambda}$ is a periodic orbit,

(2) $\Sigma_{\lambda}$ consists of infinitely many singular points, or

(3) $\Sigma_{\lambda}$ contains at most $2(k+1)=4(m+1)$ singular points, and has $4 N+n(N+n \geq 1)$ singular points, $2 N$ of which are saddle points, $2 N$ of which are stable node points (possibly degenerate), and $n$ of which have index zero.

3.3. Proof of Theorem 1.1. In this subsection, we shall prove the first result which is related to the classification of the structure of $\Sigma_{R}$.

STEP 1.We divide the proof into several steps. In the first step, we shall consider the eigenvalue problem of the linearized equation of (2.1) and shall find the eigenvectors and the critical Rayleigh number $R_{c}$.

Let's consider the eigenvalue problem of the linear equation,

$$
L_{\lambda} T=\beta(\lambda) T \text {. }
$$

It is equivalent to

$$
-\Delta T-\lambda u_{2}(T)+\beta(\lambda) T=0,
$$

where $\lambda=\sqrt{R}$. Because $u(T)$ satisfies

$$
-\Delta u(T)+\nabla p-\lambda T k=0,
$$

we can put two equations together as follows:

$$
\left\{\begin{array}{l}
-\Delta u(T)+\nabla p-\lambda T k=0, \\
-\Delta T-\lambda u_{2}(T)+\beta(\lambda) T=0, \\
\nabla \cdot u=0 .
\end{array}\right.
$$

For the no-slip boundary conditions, the following separation of variables is appropriate: for $T \in H_{1}$,

$$
\begin{aligned}
(u, T) & =\left(u_{1}, u_{2}, T\right) \\
& =\left(-\sin \frac{2 k \pi}{L} x_{1} H^{\prime}\left(x_{2}\right), \frac{2 k \pi}{L} \cos \frac{2 k \pi}{L} x_{1} H\left(x_{2}\right), \cos \frac{2 k \pi}{L} x_{1} \Theta\left(x_{2}\right)\right), \\
(u, T) & =\left(u_{1}, u_{2}, T\right) \\
& =\left(\cos \frac{2 k \pi}{L} x_{1} H^{\prime}\left(x_{2}\right), \frac{2 k \pi}{L} \sin \frac{2 k \pi}{L} x_{1} H\left(x_{2}\right), \sin \frac{2 k \pi}{L} x_{1} \Theta\left(x_{2}\right)\right) .
\end{aligned}
$$

or, 
Then, from (3.19) we can derive a system of ODEs

$$
\left\{\begin{array}{l}
\left(D^{2}-a_{k}^{2}\right)^{2} H=\lambda a_{k} \Theta, \\
\left(D^{2}-a_{k}^{2}\right) \Theta=-\lambda a_{k} H+\beta(\lambda) \Theta,
\end{array}\right.
$$

supplemented with the boundary conditions

$$
H=D H=\Theta=0 \quad \text { at } \quad x_{2}=0,1,
$$

where $D=\frac{d}{d x_{2}}$ and $a_{k}=\frac{2 k \pi}{L}$.

The eigenvalue problem (3.20) with boundary conditions (3.21) is symmetric, and has complete sequence of eigenvalues and eigenvectors for given $k \geq 0$ and $\lambda$ :

$$
\left\{\begin{array}{l}
\beta_{k, j}(\lambda) \geq \beta_{k, j+1}(\lambda), \quad \beta_{k, j} \rightarrow-\infty \text { as } j \rightarrow \infty, \\
H_{k, j} \in H^{4}(0,1) \cap H_{0}^{2}(0,1), \\
\Theta_{k, j} \in H^{2}(0,1) \cap H_{0}^{1}(0,1),
\end{array}\right.
$$

for $j=1,2, \cdots$. Moreover,

$$
\left\{H_{k, j}, \Theta_{k, j} \mid j=1,2, \cdots\right\}
$$

forms an orthogonal basis of $L^{2}(0,1) \times L^{2}(0,1)$.

From the above arguments, it can be seen that the following sequences are the set of eigenvectors of (3.19):

$$
\begin{aligned}
& \left(u_{k, 2 j-1}, T_{k, 2 j-1}\right) \\
& =\left(-\sin \frac{2 k \pi}{L} x_{1} H_{k, 2 j-1}^{\prime}\left(x_{2}\right), \frac{2 k \pi}{L} \cos \frac{2 k \pi}{L} x_{1} H_{k, 2 j-1}\left(x_{2}\right), \cos \frac{2 k \pi}{L} x_{1} \Theta_{k, 2 j-1}\left(x_{2}\right)\right),
\end{aligned}
$$

$$
\left(u_{k, 2 j}, T_{k, 2 j}\right)=\left(\cos \frac{2 k \pi}{L} x_{1} H_{k, 2 j}^{\prime}\left(x_{2}\right), \frac{2 k \pi}{L} \sin \frac{2 k \pi}{L} x_{1} H_{k, 2 j}\left(x_{2}\right), \sin \frac{2 k \pi}{L} x_{1} \Theta_{k, 2 j}\left(x_{2}\right)\right),
$$

where $k \geq 0$ and $j \geq 1$.

We know that

$$
\begin{aligned}
& \beta_{11}(\lambda)=\beta_{12}(\lambda) \begin{cases}<0 & \text { if } \lambda<\lambda_{c}, \\
=0 & \text { if } \lambda=\lambda_{c}, \\
>0 & \text { if } \lambda>\lambda_{c},\end{cases} \\
& \beta_{11}>\beta_{k j}\left(\lambda_{c}\right) \quad \text { if } \quad(k, j) \neq(1,1),
\end{aligned}
$$

so that $\beta_{k, j}\left(\lambda_{c}\right)<0$ for $(k, j) \neq(1,1)$ and the first eigenvectors of (3.19) corresponding to $\beta_{11}$ are

$$
\left(u_{11}, T_{11}\right) \text { and }\left(u_{12}, T_{12}\right)
$$


We remark here that we can derive from (3.19), (3.23) and (3.24) that for $k=0$

$$
\begin{aligned}
\left(u_{0,2 j-1}, T_{0,2 j-1}\right) & =\left(0,0, \sin j \pi x_{2}\right), \\
\left(u_{0,2 j}, T_{0,2 j}\right) & =\left(H_{0,2 j}^{\prime}\left(x_{2}\right), 0,0\right) .
\end{aligned}
$$

STEP 2.We shall show that the bifurcated attractor of (2.1) and (2.2) contains a singularity cycle and it can be shown in the exactly same way which has been used in [16]. We omit the details.

STEP 3. In the third step, we shall investigate the topological structure of $\Sigma_{R}$. Let $E_{1}^{\lambda}=E_{0}=\operatorname{span}\left\{T_{11}, T_{12}\right\}$ and $E_{2}^{\lambda}=E_{0}^{\perp}$. For $T \in H$, we have the Furier expansion

$$
T=\sum_{k \geq 0, j \geq 1} y_{k j} T_{k, j}
$$

Then the reduction equations of (2.8) are as follows:

$$
\left\{\begin{array}{l}
\frac{d y_{11}}{d t}=\beta_{11}(\lambda) y_{11}+\frac{1}{<T_{11}, T_{11}>_{H}}<G_{2}(T, T), T_{11}>_{H} \\
\frac{d y_{12}}{d t}=\beta_{11}(\lambda) y_{12}+\frac{1}{<T_{12}, T_{12}>_{H}}<G_{2}(T, T), T_{12}>_{H}
\end{array}\right.
$$

where $G_{2}$ is the bilinear operator of $G$ such that

$$
\begin{aligned}
G_{2}(T, T) & =-(u(T) \cdot \nabla) T \\
<G_{2}\left(T_{1}, T_{2}\right), T_{3}>_{H} & =-\int_{\Omega}\left(u\left(T_{1}\right) \cdot \nabla T_{2}\right) T_{3} d x .
\end{aligned}
$$

Let $\Phi$ be the center manifold function. Then

$$
T=y_{11} T_{11}+y_{12} T_{12}+\Phi .
$$

We note here that for $T_{11}$ and $T_{12}$,

$$
\begin{aligned}
<G_{2}\left(T_{1}, T_{2}\right), T_{3}>_{H} & =-\int_{\Omega}\left(u\left(T_{1}\right) \cdot \nabla T_{2}\right) T_{3} d x \\
& =-<G_{2}\left(T_{1}, T_{3}\right), T_{2}>_{H} .
\end{aligned}
$$

In particular,

$$
<G_{2}\left(T_{1}, T_{2}\right), T_{2}>_{H}=0 .
$$

Therefore, we have

$$
\begin{aligned}
& <G_{2}(T, T), T_{11}>_{H} \\
& \quad=<G_{2}\left(T_{11}, T_{12}\right), T_{11}>_{H} y_{11} y_{12}+<G_{2}\left(T_{11}, \Phi\right), T_{11}>_{H} y_{11} \\
& \quad+<G_{2}\left(T_{12}, T_{12}\right), T_{11}>_{H} y_{12}^{2}+<G_{2}\left(T_{12}, \Phi\right), T_{11}>_{H} y_{12} \\
& \quad+<G_{2}\left(\Phi, T_{12}\right), T_{11}>_{H} y_{12}+<G_{2}(\Phi, \Phi), T_{11}>_{H},
\end{aligned}
$$


and

$$
\begin{aligned}
& <G_{2}(T, T), T_{12}>_{H} \\
& \quad=<G_{2}\left(T_{11}, T_{11}\right), T_{12}>_{H} y_{11}^{2}+<G_{2}\left(T_{11}, \Phi\right), T_{12}>_{H} y_{11} \\
& \quad+<G_{2}\left(T_{12}, T_{11}\right), T_{12}>_{H} y_{11} y_{12}+<G_{2}\left(T_{12}, \Phi\right), T_{12}>_{H} y_{12} \\
& \quad+<G_{2}\left(\Phi, T_{11}\right), T_{12}>_{H} y_{11}+<G_{2}(\Phi, \Phi), T_{12}>_{H} .
\end{aligned}
$$

It is easy to check that the following inner products vanish for $k \geq 0, j \geq 1$

$$
\begin{aligned}
& <G_{2}\left(T_{11}, T_{12}\right), T_{k, 2 j-1}>_{H} \\
& =\frac{2 \pi}{L} \int_{0}^{L} \int_{0}^{1}\left[H_{11}^{\prime}\left(x_{2}\right) \Theta_{12}\left(x_{2}\right) \Theta_{k, 2 j-1}\left(x_{2}\right)-H_{11}\left(x_{2}\right) \Theta_{12}^{\prime}\left(x_{2}\right) \Theta_{k, 2 j-1}\left(x_{2}\right)\right] \\
& =0, \quad \sin \frac{2 \pi}{L} x_{1} \cos \frac{2 \pi}{L} x_{1} \cos \frac{2 k \pi}{L} x_{1} d x \\
& <G_{2}\left(T_{12}, T_{11}\right), T_{k, 2 j-1}>_{H} \\
& =\frac{2 \pi}{L} \int_{0}^{L} \int_{0}^{1}\left[H_{12}^{\prime}\left(x_{2}\right) \Theta_{11}\left(x_{2}\right) \Theta_{k, 2 j-1}\left(x_{2}\right)-H_{12}\left(x_{2}\right) \Theta_{11}^{\prime}\left(x_{2}\right) \Theta_{k, 2 j-1}\left(x_{2}\right)\right] \\
& =0, \quad \sin \frac{2 \pi}{L} x_{1} \cos \frac{2 \pi}{L} x_{1} \cos \frac{2 k \pi}{L} x_{1} d x \\
& <G_{2}\left(T_{11}, T_{11}\right), T_{k, 2 j}>_{H} \\
& =-\frac{2 \pi}{L} \int_{0}^{L} \int_{0}^{1}\left(\sin \frac{2 \pi}{L} x_{1}\right)^{2} \sin \frac{2 k \pi}{L} x_{1} H_{11}^{\prime}\left(x_{2}\right) \Theta_{11}\left(x_{2}\right) \Theta_{k, 2 j}\left(x_{2}\right) \\
& \quad+\left(\cos \frac{2 \pi}{L} x_{1}\right)^{2} \sin \frac{2 k \pi}{L} x_{1} H_{11}\left(x_{2}\right) \Theta_{11}^{\prime}\left(x_{2}\right) \Theta_{k, 2 j}\left(x_{2}\right) d x \\
& <G_{2}\left(T_{12}, T_{12}\right), T_{k, 2 j}>_{H} \\
& =-\frac{2 \pi}{L} \int_{0}^{L} \int_{0}^{1}\left(\cos \frac{2 \pi}{L} x_{1}\right)^{2} \sin \frac{2 k \pi}{L} x_{1} H_{12}^{\prime}\left(x_{2}\right) \Theta_{12}\left(x_{2}\right) \Theta_{k, 2 j}\left(x_{2}\right) \\
& =0, \quad+\left(\sin \frac{2 \pi}{L} x_{1}\right)^{2} \sin \frac{2 k \pi}{L} x_{1} H_{12}\left(x_{2}\right) \Theta_{12}^{\prime}\left(x_{2}\right) \Theta_{k, 2 j}\left(x_{2}\right) d x \\
& \quad=0, \quad(x)
\end{aligned}
$$




$$
\begin{aligned}
& <G_{2}\left(T_{k, 2 j-1}, T_{12}\right), T_{11}>_{H} \\
& =\int_{0}^{L} \int_{0}^{1} \frac{2 \pi}{L} \sin \frac{2 k \pi}{L} x_{1}\left(\cos \frac{2 \pi}{L} x_{1}\right)^{2} H_{k, 2 j-1}^{\prime}\left(x_{2}\right) \Theta_{12}\left(x_{2}\right) \Theta_{11}\left(x_{2}\right) \\
& \quad-\frac{2 k \pi}{L} \cos \frac{2 k \pi}{L} x_{1} \sin \frac{2 \pi}{L} x_{1} \cos \frac{2 \pi}{L} x_{1} H_{k, 2 j-1}\left(x_{2}\right) \Theta_{12}^{\prime}\left(x_{2}\right) \Theta_{11}\left(x_{2}\right) d x \\
& =0
\end{aligned}
$$

We also have

$$
\begin{aligned}
& <G_{2}\left(T_{11}, T_{11}\right), T_{k, 2 j-1}>_{H} \\
& =-\frac{2 \pi}{L} \int_{0}^{L} \int_{0}^{1}\left(\sin \frac{2 \pi}{L} x_{1}\right)^{2} \cos \frac{2 k \pi}{L} x_{1} H_{11}^{\prime}\left(x_{2}\right) \Theta_{11}\left(x_{2}\right) \Theta_{k, 2 j-1}\left(x_{2}\right) \\
& +\left(\cos \frac{2 \pi}{L} x_{1}\right)^{2} \cos \frac{2 k \pi}{L} x_{1} H_{11}\left(x_{2}\right) \Theta_{11}^{\prime}\left(x_{2}\right) \Theta_{k, 2 j-1}\left(x_{2}\right) d x \\
& = \begin{cases}\neq 0 & \text { if } \quad k=0,2 \\
=0 & \text { otherwise }\end{cases} \\
& <G_{2}\left(T_{12}, T_{12}\right), T_{k, 2 j-1}>_{H} \\
& =-\frac{2 \pi}{L} \int_{0}^{L} \int_{0}^{1}\left(\cos \frac{2 \pi}{L} x_{1}\right)^{2} \cos \frac{2 k \pi}{L} x_{1} H_{12}^{\prime}\left(x_{2}\right) \Theta_{12}\left(x_{2}\right) \Theta_{k, 2 j-1}\left(x_{2}\right) \\
& +\left(\sin \frac{2 \pi}{L} x_{1}\right)^{2} \cos \frac{2 k \pi}{L} x_{1} H_{12}\left(x_{2}\right) \Theta_{12}^{\prime}\left(x_{2}\right) \Theta_{k, 2 j-1}\left(x_{2}\right) d x \\
& = \begin{cases}\neq 0 & \text { if } \quad k=0,2 \\
=0 & \text { otherwise }\end{cases} \\
& \begin{array}{l}
<G_{2}\left(T_{11}, T_{12}\right), T_{k, 2 j}>_{H} \\
=\frac{2 \pi}{L} \int_{0}^{L} \int_{0}^{1} \sin \frac{2 \pi}{L} x_{1} \cos \frac{2 \pi}{L} x_{1} \sin \frac{2 k \pi}{L} x_{1} H_{11}^{\prime}\left(x_{2}\right) \Theta_{12}\left(x_{2}\right) \Theta_{k, 2 j}\left(x_{2}\right) \\
\quad-\cos \frac{2 \pi}{L} x_{1} \sin \frac{2 \pi}{L} x_{1} \sin \frac{2 k \pi}{L} x_{1} H_{11}\left(x_{2}\right) \Theta_{12}^{\prime}\left(x_{2}\right) \Theta_{k, 2 j}\left(x_{2}\right) d x \\
= \begin{cases}\neq 0 & \text { if } k=2, \\
=0 & \text { otherwise, }\end{cases}
\end{array}
\end{aligned}
$$




$$
\begin{aligned}
& <G_{2}\left(T_{12}, T_{11}\right), T_{k, 2 j}>_{H} \\
& =\frac{2 \pi}{L} \int_{0}^{L} \int_{0}^{1} \sin \frac{2 \pi}{L} x_{1} \cos \frac{2 \pi}{L} x_{1} \sin \frac{2 k \pi}{L} x_{1} H_{12}^{\prime}\left(x_{2}\right) \Theta_{11}\left(x_{2}\right) \Theta_{k, 2 j}\left(x_{2}\right) \\
& \quad-\cos \frac{2 \pi}{L} x_{1} \sin \frac{2 \pi}{L} x_{1} \sin \frac{2 k \pi}{L} x_{1} H_{12}\left(x_{2}\right) \Theta_{11}^{\prime}\left(x_{2}\right) \Theta_{k, 2 j}\left(x_{2}\right) d x \\
& = \begin{cases}\neq 0 & \text { if } \quad k=2, \\
=0 & \text { otherwise, }\end{cases} \\
& =-G_{2}\left(T_{k, 2 j}, T_{12}\right), T_{11}>_{H} \int_{0}^{1} \frac{2 \pi}{L} \cos \frac{2 k \pi}{L} x_{1}\left(\cos \frac{2 \pi}{L} x_{1}\right)^{2} H_{k, 2 j}^{\prime}\left(x_{2}\right) \Theta_{12}\left(x_{2}\right) \Theta_{11}\left(x_{2}\right) \\
& \quad+\frac{2 k \pi}{L} \sin \frac{2 k \pi}{L} x_{1} \sin \frac{2 \pi}{L} x_{1} \cos \frac{2 \pi}{L} x_{1} H_{k, 2 j}\left(x_{2}\right) \Theta_{12}^{\prime}\left(x_{2}\right) \Theta_{11}\left(x_{2}\right) d x \\
& = \begin{cases}\neq 0 & \text { if } \quad k=0,2, \\
=0 & \text { otherwise. }\end{cases}
\end{aligned}
$$

By Theorem 3.2, it is seen that the center manifold function $\Phi$, near $\lambda=\lambda_{c}$, can be expressed as

$$
\Phi(y)=\sum_{(k, j) \neq(1,1),(1,2)} \Phi_{k, j}(y) T_{k, j}+o\left(|y|^{2}\right),
$$

where

$$
\begin{aligned}
& \Phi_{k, j}(y)=-\frac{1}{\beta_{k j}(\lambda)} \sum_{1 \leq p, q \leq 2} a_{p q}^{k j} y_{1 p} y_{1 q} \\
& a_{p q}^{k j}=<G_{2}\left(T_{1 p}, T_{1 q}\right), T_{k, j}>_{H} .
\end{aligned}
$$

Due to the above inner products and (3.28), (3.26) and (3.27) become

$$
\begin{aligned}
<G_{2}(T, T), T_{11}>_{H} \\
=-\sum_{j=2}^{\infty}\left[<G_{2}\left(T_{11}, T_{11}\right), T_{0,2 j-1}>_{H} \Phi_{0,2 j-1}\right. \\
\left.\quad+<G_{2}\left(T_{11}, T_{11}\right), T_{2,2 j-1}>_{H} \Phi_{2,2 j-1}\right] y_{11} \\
\quad-\sum_{j=2}^{\infty}\left[<G_{2}\left(T_{12}, T_{11}\right), T_{2,2 j}>_{H} \Phi_{2,2 j}\right] y_{12} \\
+\sum_{j=2}^{\infty}\left[<G_{2}\left(T_{0,2 j}, T_{12}\right), T_{11}>_{H} \Phi_{0,2 j}\right. \\
\left.\quad+<G_{2}\left(T_{2,2 j}, T_{12}\right), T_{11}>_{H} \Phi_{2,2 j}\right] y_{12} \\
+<G_{2}(\Phi, \Phi), T_{11}>_{H},
\end{aligned}
$$


and

$$
\begin{aligned}
<G_{2}(T, T), T_{12}>_{H} \\
=-\sum_{j=2}^{\infty}\left[<G_{2}\left(T_{12}, T_{12}\right), T_{0,2 j-1}>_{H} \Phi_{0,2 j-1}\right. \\
\left.\quad+<G_{2}\left(T_{12}, T_{12}\right), T_{2,2 j-1}>_{H} \Phi_{2,2 j-1}\right] y_{12} \\
\quad-\sum_{j=2}^{\infty}\left[<G_{2}\left(T_{11}, T_{12}\right), T_{2,2 j}>_{H} \Phi_{2,2 j}\right] y_{11} \\
+\sum_{j=2}^{\infty}\left[<G_{2}\left(T_{0,2 j}, T_{11}\right), T_{12}>_{H} \Phi_{0,2 j}\right. \\
\left.\quad+<G_{2}\left(T_{2,2 j}, T_{11}\right), T_{12}>_{H} \Phi_{2,2 j}\right] y_{11} \\
+<G_{2}(\Phi, \Phi), T_{12}>_{H} .
\end{aligned}
$$

Direct calculations yield

$$
\begin{aligned}
<G_{2}\left(T_{2,2 j}, T_{12}\right), T_{11}>_{H} & =-\int_{\Omega}\left(u\left(T_{2,2 j} \cdot \nabla T_{12}\right) T_{1} 1 d x\right. \\
& =-\frac{\pi}{2} \int_{0}^{1}\left(H_{2,2 j}^{\prime} \Theta_{12} \Theta_{11}+2 H_{2,2 j} \Theta_{12}^{\prime} \Theta_{11}\right) d x_{2} \\
& =-\frac{\pi}{2} \int_{0}^{1} \frac{d}{d x_{2}}\left(H_{2,2 j} \Theta_{11}^{2}\right) d x_{2} \\
& =0
\end{aligned}
$$

and some identities

$$
\begin{aligned}
<G_{2}\left(T_{11}, T_{11}\right), T_{0,2 j-1}>_{H} & =<G_{2}\left(T_{12}, T_{12}\right), T_{0,2 j-1}>_{H}, \\
<G_{2}\left(T_{11}, T_{12}\right), T_{2,2 j}>_{H} & =<G_{2}\left(T_{12}, T_{11}\right), T_{2,2 j}>_{H}, \\
<G_{2}\left(T_{11}, T_{11}\right), T_{2,2 j-1}>_{H} & =-<G_{2}\left(T_{12}, T_{12}\right), T_{2,2 j-1}>_{H} .
\end{aligned}
$$


By (3.28), near $\lambda=\lambda_{c}$ we can calculate coefficients of the center manifold function explicitly:

$$
\begin{aligned}
\Phi_{0,2 j-1} & =-\frac{1}{\beta_{0,2 j-1}(\lambda)} \sum_{1 \leq p, q \leq 2} a_{p q}^{0,2 j-1} y_{1 p} y_{1 q} \\
& =-\frac{1}{\beta_{0,2 j-1}(\lambda)}<G_{2}\left(T_{11}, T_{11}\right), T_{0,2 j-1}>_{H}\left(y_{11}^{2}+y_{12}^{2}\right) \\
& +o\left(\left|y_{11}\right|^{2}+\left|y_{12}\right|^{2}\right) \\
\Phi_{2,2 j-1} & =-\frac{1}{\beta_{2,2 j-1}(\lambda)} \sum_{1 \leq p, q \leq 2} a_{p q}^{2,2 j-1} y_{1 p} y_{1 q} \\
& =-\frac{1}{\beta_{2,2 j-1}(\lambda)}<G_{2}\left(T_{11}, T_{11}\right), T_{2,2 j-1}>_{H}\left(y_{11}^{2}-y_{12}^{2}\right) \\
& +o\left(\left|y_{11}\right|^{2}+\left|y_{12}\right|^{2}\right) \\
\Phi_{0,2 j} & =-\frac{1}{\beta_{0,2 j}(\lambda)} \sum_{1 \leq p, q \leq 2} a_{p q}^{0,2 j} y_{1 p} y_{1 q} \\
& =0 \\
\Phi_{2,2 j} & =-\frac{1}{\beta_{2,2 j}(\lambda)} \sum_{1 \leq p, q \leq 2} a_{p q}^{2,2 j} y_{1 p} y_{1 q} \\
& =-\frac{2}{\beta_{2,2 j}(\lambda)}<G_{2}\left(T_{11}, T_{12}\right), T_{2,2 j}>_{H} y_{11} y_{12} \\
& +o\left(\left|y_{11}\right|^{2}+\left|y_{12}\right|^{2}\right) .
\end{aligned}
$$

Replacing (3.29) and (3.30) with (3.31), we obtain

$$
\begin{aligned}
<G_{2} & (T, T), T_{11}>_{H} \\
= & -\sum_{j=2}^{\infty}\left(\frac{1}{\beta_{0,2 j-1}(\lambda)}<G_{2}\left(T_{11}, T_{11}\right), T_{0,2 j-1}>_{H}^{2}\right) y_{11}\left(y_{11}^{2}+y_{12}^{2}\right) \\
& -\sum_{j=2}^{\infty}\left(\frac{1}{\beta_{2,2 j-1}(\lambda)}<G_{2}\left(T_{11}, T_{11}\right), T_{2,2 j-1}>_{H}^{2}\right) y_{11}\left(y_{11}^{2}+y_{12}^{2}\right) \\
& +<G_{2}(\Phi, \Phi), T_{11}>_{H}+o\left(\left|y_{11}\right|^{3}+\left|y_{12}\right|^{3}\right),
\end{aligned}
$$

and

$$
\begin{aligned}
<G_{2} & (T, T), T_{12}>_{H} \\
= & -\sum_{j=2}^{\infty}\left(\frac{1}{\beta_{0,2 j-1}(\lambda)}<G_{2}\left(T_{11}, T_{11}\right), T_{0,2 j-1}>_{H}^{2}\right) y_{12}\left(y_{11}^{2}+y_{12}^{2}\right) \\
& -\sum_{j=2}^{\infty}\left(\frac{1}{\beta_{2,2 j-1}(\lambda)}<G_{2}\left(T_{11}, T_{11}\right), T_{2,2 j-1}>_{H}^{2}\right) y_{12}\left(y_{11}^{2}+y_{12}^{2}\right) \\
& +<G_{2}(\Phi, \Phi), T_{12}>_{H}+o\left(\left|y_{11}\right|^{3}+\left|y_{12}\right|^{3}\right) .
\end{aligned}
$$


We know that the center manifold function involves only higher order terms

which implies that

$$
\Phi(y)=O\left(\left|y_{11}\right|^{2}+\left|y_{12}\right|^{2}\right),
$$

$$
<G_{2}(\Phi, \Phi), T_{1 i}>_{H}=o\left(\left|y_{11}\right|^{3},\left|y_{12}\right|^{3}\right), \quad(i=1,2) .
$$

Let

$$
\alpha=\sum_{j=2}^{\infty}\left(\frac{<G_{2}\left(T_{11}, T_{11}\right), T_{0,2 j-1}>_{H}^{2}}{\beta_{0,2 j-1}(\lambda)}+\frac{<G_{2}\left(T_{11}, T_{11}\right), T_{2,2 j-1}>_{H}^{2}}{\beta_{2,2 j-1}(\lambda)}\right) .
$$

Then $\alpha<0$ for $\lambda_{c}<\lambda<\lambda_{c}+\varepsilon$. Note that for $k \geq 0$ and $j \geq 1$,

$$
\left\|T_{k, 2 j-1}\right\|_{H}^{2}=\left\|T_{k, 2 j}\right\|_{H}^{2} \text {. }
$$

Then, from (3.34) we get the bifurcation equations reduced on the center manifold:

$$
\begin{aligned}
& \frac{d y_{11}}{d t}=\beta_{11}(\lambda) y_{11}+\frac{\alpha}{\left\|T_{k, 2 j-1}\right\|_{H}^{2}} y_{11}\left(y_{11}^{2}+y_{12}^{2}\right)+o\left(\left|y_{11}\right|^{3},\left|y_{12}\right|^{3}\right), \\
& \frac{d y_{12}}{d t}=\beta_{11}(\lambda) y_{12}+\frac{\alpha}{\|\left. T_{k, 2 j}\right|_{H} ^{2}} y_{12}\left(y_{11}^{2}+y_{12}^{2}\right)+o\left(\left|y_{11}\right|^{3},\left|y_{12}\right|^{3}\right) .
\end{aligned}
$$

It can be easily verified that (3.35) satisfies conditions of Theorem 3.7 so that we can conclude that $\Sigma_{R}$ is homeomorphic to $S^{1}$.

This completes the proof.

\section{Structural Stability Theorems}

4.1. Basic Settings. In this section we shall recall some results on structural stability for two-dimensional divergence free vector fields governed by the Navier-Stokes equations or the Euler equations. see $[11,13]$ for details.

Let $C^{r}\left(\Omega, \mathbb{R}^{2}\right)$ be the space of all $C^{r}(r \geq 1)$ vector fields on $\Omega$, which are periodic in $x_{1}$ direction with periods $L$, and let

$$
\begin{aligned}
& D^{r}\left(\Omega, \mathbb{R}^{2}\right)=\left\{v \in C^{r}\left(\Omega, \mathbb{R}^{2}\right) \mid \nabla \cdot v=0, v_{2}=0 \text { at } x_{2}=0,1\right\}, \\
& B_{0}^{r}\left(\Omega, \mathbb{R}^{2}\right)=\left\{v \in D^{r}\left(\Omega, \mathbb{R}^{2}\right) \mid v=0 \text { at } x_{2}=0,1\right\} .
\end{aligned}
$$

Definition 4.1. Two vector fields $u, v \in C^{r}\left(\Omega, \mathbb{R}^{2}\right)$ are called topologically equivalent if there exists a homeomorphism of $\varphi: \Omega \rightarrow \Omega$, which takes the orbits of $u$ to that of $v$ and preserves their orientations.

Definition 4.2. Let $X=D^{r}\left(\Omega, \mathbb{R}^{2}\right)$. A vector field $v \in X$ is called structurally stable in $X$ if there exists a neighborhood $U \subset X$ of $v$ such that for any $u \in U, u$ and $v$ are topologically equivalent.

Let $v \in D^{r}\left(\Omega, \mathbb{R}^{2}\right)$. We recall some basic definitions and properties on divergence free vector fields.

1. A point $p \in \Omega$ is called a singular point of $v$ if $v(p)=0$; a singular point $p$ of $v$ is called non-degenerate if the Jacobian matrix $D v(p)$ is invertible; $v$ is called regular if all singular points of $v$ are non-degenerate. 
2. An interior non-degenerate singular point of $v$ can be either a center or a saddle, and a non-degenerate boundary singular point must be a saddle.

3. Saddles of $v$ must be connected to saddles. An interior saddle $p \in \Omega$ is called self connected if $p$ is connected to itself. i.e., $p$ occurs in a graph whose topological form is that of the ' $\infty$ '.

Let $v \in B_{0}^{r}\left(\Omega, \mathbb{R}^{2}\right)$. Then, it is obvious that each point on $x_{2}=0,1$ is a singular point of $v$. Now, we need to classify the boundary points of $\Omega$.

Definition 4.3. Let $u \in B_{0}^{r}(\Omega, \mathbb{R})(r \geq 2)$.

1. A point $p \in \partial \Omega$ is called a $\partial$-regular point of $u$ if $\frac{\partial u_{\tau}(p)}{\partial n} \neq 0$; otherwise, $p$ is called a $\partial$-singular point of $u$.

2. A $\partial$-singular point $p \in \partial \Omega$ of $u$ is called non-degenerate if

$$
\operatorname{det}\left(\begin{array}{ll}
\frac{\partial^{2} u_{\tau}(p)}{\partial \tau \partial n} & \frac{\partial^{2} u_{\tau}(p)}{\partial n^{2}} \\
\frac{\partial^{2} u_{n}(p)}{\partial \tau \partial n} & \frac{\partial^{2} u_{n}(p)}{\partial n^{2}}
\end{array}\right) \neq 0 .
$$

A non-degenerate $\partial$-singular point of $u$ is also called a $\partial$-saddle point of $u$.

3. $u$ is called $D$-regular if $u$ is regular in int $\Omega$, and all $\partial$-singular points of $u$ on $\partial \Omega$ are non-degenerate.

Lemma 4.4. (Connection Lemma, [13]) Let $u, v \in D^{r}\left(\Omega, \mathbb{R}^{2}\right)$ with $v$ sufficiently small, and $L \subset \Omega$ be an extended orbit of $u$ starting at $p$. Then an extended orbit $\gamma$ of $u+v$ starting at $p$ passes through a point $q \in L$ if and only if

$$
\int_{L[p, q]} v \times d \ell=0
$$

where $L[p, q]$ is the curve segment on $L$ from $p$ and $q$.

Theorem 4.5. $[11,13]$ Let $u \in B_{0}^{r}\left(\Omega, \mathbb{R}^{2}\right)(r \geq 2)$. Then $u$ is structurally stable if and only if

(1) $u$ is D-regular,

(2) all interior saddle points of $u$ are self-connected, and

(3) each $\partial$-saddle point of $u$ on $\partial \Omega$ is connected to a $\partial$-saddle points on the same connected component of $\partial \Omega$.

Remark 4.6. The difference between two boundary conditions, no-slip and no-stress, is due to the zero-average condition. Since $((\alpha, 0), 0)$ is a solution of (1.10)-(1.12) for any constant $\alpha$ under no-stress boundary condition, the velocity field in the function space must satisfy $\int_{\Omega} u d x=0$. Hence, an orbit connecting two saddles on different components of the boundary can not be broken with a perturbation into orbits connecting only saddles on the same connected component of the boundary. see [16] for details.

Now, we prove the structural stability theorem. 
4.2. Proof of Theorem 1.2. We infer from (3.35) that for any $T \in \Sigma_{R}$, the associated steady state velocity field $u=\left(u_{1}, u_{2}\right)$ can be expressed as

$$
u=\sum_{k=1}^{2} y_{1 k} u_{1 k}+o\left(\sum_{k=1}^{2} y_{1 k} u_{1 k}\right),
$$

which implies that

$$
\begin{aligned}
& u_{1}=y_{11}\left(-\sin \frac{2 \pi}{L} x_{1} H_{11}^{\prime}\right)+y_{12}\left(\cos \frac{2 \pi}{L} x_{1} H_{11}^{\prime}\right)+v_{1}\left(y_{11}, y_{12}, \beta_{11}\right), \\
& u_{2}=y_{11}\left(\frac{2 \pi}{L} \cos \frac{2 \pi}{L} x_{1} H_{11}\right)+y_{12}\left(\frac{2 \pi}{L} \sin \frac{2 \pi}{L} x_{1} H_{11}\right)+v_{2}\left(y_{11}, y_{12}, \beta_{11}\right) .
\end{aligned}
$$

Now, note that the above expansion were done in terms of the eigenvectors. Thus, they must hold not only on $H$, but also on higher dimensional Sobolev spaces $H^{m}(m \geq 0)$. Simple modifications yield

$$
\begin{aligned}
& u_{1}=r \sin \frac{2 \pi}{L}\left(\theta-x_{1}\right) H_{11}^{\prime}+v_{1}\left(y_{11}, y_{12}, \beta_{11}\right), \\
& u_{2}=\frac{2 \pi r}{L} \cos \frac{2 \pi}{L}\left(\theta-x_{1}\right) H_{11}+v_{2}\left(y_{11}, y_{12}, \beta_{11}\right),
\end{aligned}
$$

for some $0 \leq \theta \leq 2 \pi$. Here,

$$
\begin{gathered}
r^{2}=y_{11}^{2}+y_{12}^{2}=\frac{\beta_{11}(\lambda)}{-\alpha}\left\|T_{11}\right\|_{H}^{2}+o\left(\left|\frac{\beta(\lambda)}{-\alpha}\right|\right), \text { for } \lambda>\lambda_{11}, \\
v_{i}\left(y_{11}, y_{12}, \beta_{11}\right)=o(r) \quad(i=1,2) .
\end{gathered}
$$

Let

$$
E=\left\{(u, T) \in \widetilde{H} \times H \mid \int_{\Omega} u_{1} d x=0\right\} .
$$

Then, $E$ is invariant for the equations (1.10)-(1.12), which implies that

$$
\int_{0}^{1} v_{1} d x_{2}=0
$$

Therefore, in the Fourier expansion of $u$ in (3.23) and (3.24), the coefficients of $\left(u_{0,2 j}, T_{0,2 j}\right)=\left(H_{0,2 j}^{\prime}\left(x_{2}\right), 0,0\right)$ are zero. By the Connection Lemma, it follows that $u=\left(u_{1}, u_{2}\right)$ is topologically equivalent to the velocity field

$$
u^{0}=\left(u_{1}^{0}, u_{2}^{0}\right)=\left(r \sin \frac{2 \pi}{L} x_{1} H_{11}^{\prime}\left(x_{2}\right), \frac{2 \pi}{L} \cos \frac{2 \pi}{L} x_{1} H_{11}\left(x_{2}\right)\right) .
$$

It is known that the first eigenvector $H_{11}\left(x_{2}\right)$ at $\lambda=\lambda_{c}$ is given by

$$
\begin{aligned}
H_{11}\left(x_{2}\right)= & \cos q_{0}\left(x_{2}-\frac{1}{2}\right)-0.06151664 \cosh q_{1}\left(x_{2}-\frac{1}{2}\right) \cos q_{2}\left(x-\frac{1}{2}\right) \\
& +0.103887 \sinh q_{1}\left(x_{2}-\frac{1}{2}\right) \sin q_{2}\left(x_{2}-\frac{1}{2}\right)
\end{aligned}
$$

where $q_{0}=3.973639 ; q_{1}=5.095214 ; q_{2}=2.126096$; see [2] for details. The structure of $u^{0}$, the steady state solution, is pure roll structure whose flow, 
on the top and bottom plates, does not move and is as shown in Figure 4.1. Now it suffices to show that $u^{0}$ is structurally stable.

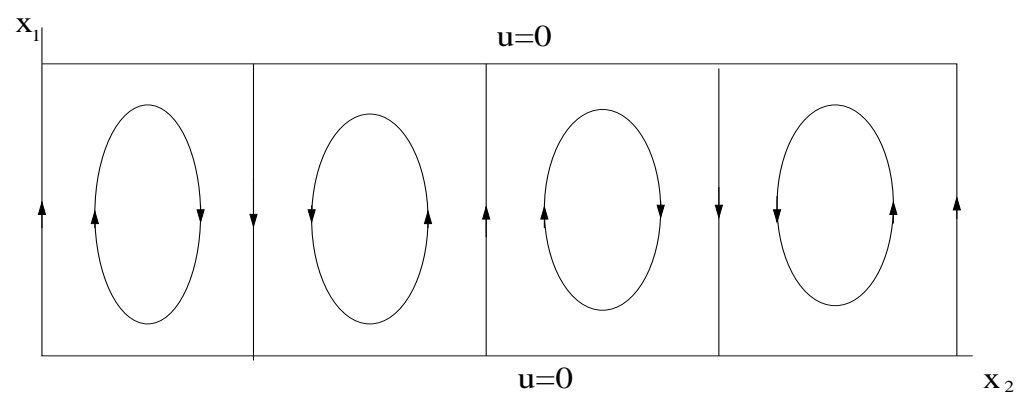

Figure 4.1. Schematic Flow Structure of $u^{0}$ with no-slip boundary condition which means that the flow does not move on the top and bottom boundaries.

From

$$
\frac{\partial u_{1}^{0}}{\partial x_{2}}=r \sin \frac{2 \pi}{L} x_{1} H^{\prime \prime}\left(x_{2}\right)=0
$$

the $\partial$-singular points of $u$ are

$$
\left(x_{1}, x_{2}\right)=\left(\frac{k L}{2}, 0\right),\left(\frac{k L}{2}, 1\right),
$$

and we can see that

$$
\begin{aligned}
\operatorname{det}\left[\begin{array}{cc}
\frac{\partial^{2} u_{1}^{0}}{\partial x_{1} \partial x_{2}} & \frac{\partial^{2} u_{1}^{0}}{\partial x_{2}^{2}} \\
\frac{\partial^{2} u_{2}^{0}}{\partial x_{1} \partial x_{2}} & \frac{\partial^{2} u_{2}^{0}}{\partial x_{2}^{2}}
\end{array}\right] & =\operatorname{det}\left[\begin{array}{cc}
\frac{2 \pi r}{L} \cos \frac{2 \pi}{L} x_{1} H_{11}^{\prime \prime}\left(x_{2}\right) & r \sin \frac{2 \pi}{L} x_{1} H_{11}^{(3)}\left(x_{2}\right) \\
-\left(\frac{2 \pi r}{L}\right)^{2} r \sin \frac{2 \pi}{L} x_{1} H_{11}^{\prime \prime}\left(x_{2}\right) & \frac{2 \pi r}{L} \cos \frac{2 \pi}{L} x_{1} H_{11}^{\prime \prime}\left(x_{2}\right)
\end{array}\right] \\
& \neq 0,
\end{aligned}
$$

at $\left(x_{1}, x_{2}\right)=\left(\frac{k L}{2}, 0\right)$ and $\left(\frac{k L}{2}, 1\right)$. Therefore, every $\partial$-singular point of $u^{0}$ is non-degenerate. For interior singular points of $u^{0}$,

$$
\operatorname{det} D u^{0}=\operatorname{det}\left[\begin{array}{cc}
\frac{2 \pi r}{L} \cos \frac{2 \pi}{L} x_{1} H_{11}^{\prime}\left(x_{2}\right) & r \sin \frac{2 \pi}{L} x_{1} H_{11}^{\prime \prime}\left(x_{2}\right) \\
-\left(\frac{2 \pi r}{L}\right)^{2} r \sin \frac{2 \pi}{L} x_{1} H_{11}\left(x_{2}\right) & \frac{2 \pi r}{L} \cos \frac{2 \pi}{L} x_{1} H_{11}^{\prime}\left(x_{2}\right)
\end{array}\right] \neq 0 .
$$

Therefore, the velocity field $u^{0}$ is $D$-regular, which means that $u$ is $D$-regular for $\lambda_{c}<\lambda<\lambda_{c}+\varepsilon$. By Theorem 4.5, $u$ is structurally stable.

The rest part of the proof will be dedicated to show the structure of the velocity field $u$ in physical space.

For some initial value $\Psi_{0}=\left(u_{0}, T_{0}\right) \in(\widetilde{H} \times H) / E$,

$$
\Psi_{0}=\sum_{k} \alpha_{k} \Psi_{k}+\Phi_{0}
$$


where $\Phi_{0} \in E$ and $\Psi_{k}=\left(\sin \frac{k \pi}{L} x_{2}, 0,0\right), k=2 m+1,(m=0,1, \cdots)$. Since

$$
<G_{2}(T, T), \Psi_{k}>_{H}=0,
$$

the solution $\Psi=\left(u\left(t, \Psi_{0}\right), T\left(t, \Psi_{0}\right)\right)$ of $(1.10)-(1.12)$ is

$$
\Psi\left(t, \Psi_{0}\right)=\sum_{k} \alpha_{k} e^{\beta_{0 k}(\lambda) t} \Psi_{k}+\widetilde{\Phi}_{0}\left(t, \Psi_{0}\right) .
$$

By [7], we know that the solution $(u, T)$ is analytic in time and all $H^{m}$ norms of $\left(u\left(t, \Psi_{0}\right), T\left(t, \Psi_{0}\right)\right)$ remains uniformly bounded in time for $t \geq \delta>$ 0 . Therefore by Theorem 3.5, we have

$$
\lim _{t \rightarrow \infty} \min _{\phi \in \Sigma_{R}}\left\|\Psi\left(t, \Psi_{0}\right)-\phi\right\|_{C^{r}}=0 .
$$
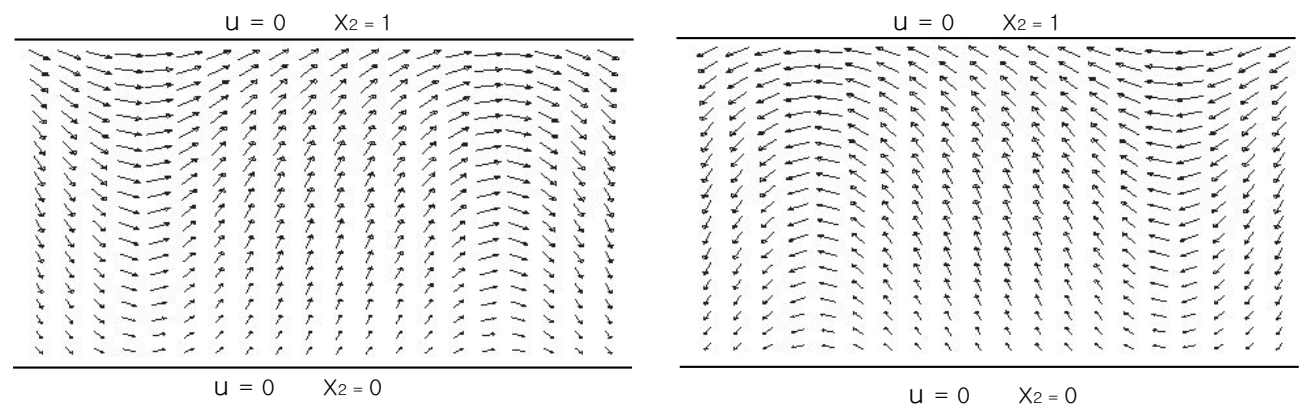

Figure 4.2. Zonally moving meandering structure of flow $u\left(t, \Psi_{0}\right)$ with no-slip boundary conditions.

Thus, $u\left(t, \Psi_{0}\right)$ is topologically equivalent to a velocity field defined by

$$
v\left(t, \Psi_{0}\right)=u^{0}+\alpha_{K} e^{\beta_{0 K}(\lambda) t}\left(\sin (K \pi / L) x_{2}, 0\right),
$$

where $K=\min \left\{k \mid \alpha_{k} \neq 0, k\right.$ is odd $\}$, and $t$ is sufficiently large. It follows from the method for breaking saddle connections $[11,13]$ that the topological structure of $v\left(t, \Psi_{0}\right)$ is as shown in Figure 4.2.

This completes the proof.

\section{REFERENCES}

[1] G. W. Branstator, A striding example of the atmosphere's leading traveling pattern, J. Atmos. sci., 44, (1987), pp. 2310-2323.

[2] S. Chandrasekhar, Hydrodynamic and Hydromagnetic Stability, Dover Publications, Inc. 1981.

[3] C. Doering And P. Constantin, Infinite prandtl number convection, J. Statistical Physics, vol.94, (1999), pp. 159-172.

[4] - On upper bounds for infinite Prandtl number convection with or without rotation, J. Mathematical Physics, vol.42, No.2, (2001), pp. 784-795.

[5] P. Drazin And W. Reid, Hydrodynamic Stability, Cambridge University Press, second ed., 2004. 
[6] R. Ecke, F. Zhong, And E. KNobloch, Hopf-Bifurcation with broken reflection symmetry in rotating Rayleigh-Bénard convection, Europhys. Lett. vo.19 (1992), pp. $177-182$.

[7] C. Foins, O. Manley, and R. Temam, Attractors for the Bénard problem: existence and physical bounds on their fractal dimension, Nonlinear Anal., 11 (1987), pp. 939967.

[8] E. Kaminski And C. JaUpart, Laminar starting plumes in high-Prandtl number fluids, J. Fluid Mech. vol.478 pp. 287-298.

[9] P. E. VAN KEKEN, Evolution of starting mantle plumes: a comparison between numerical and laboratory models, Earth. Planet. Sci. Lett., vol.148, (1997), pp. 1-11.

[10] K. KushniR, Retrograding wintertime low-frequency disturbances over the north pacific ocean, J. Atmos. sci., 44, (1987), pp. 2727-2742.

[11] T. MA AND S. WANG, Structural classification and stability of divergence-free vector fields, Physica D, 171 (2002), pp 107-126.

[12] Bifurcation Theory and Applications, World Scientific, 2005.

[13] — Geometric Theory of Incompressible Flows with Applications to Fluid Dynamics, vol. 119 of Mathematical Surveys and Monographs, Americal Mathematical Society, Providence, RI, 2005.

[14] - Rayleigh-Benard Convection: Dynamics and Structure in the Physical Space, Comm. Math. Sci., 5:3(2007), pp. 553-574

[15] J. PARK, Dynamic bifurcation theory of Rayleigh-Bénard convection with infinite prandtl number, Disc. Cont. Dyn. Sys.B, Vol.6, No.3, (2005), pp 591-604.

[16] - Two-dimensional infinite Prandtl number convection: Structure of bifurcated solutions, J. Nonlinear Sci., Vol.17, (2007), pp 199-220.

[17] A. PAZY, Semigroups of linear operators and applications to partial differential equations, vol. 44 of Applied Mathematical Sciences, Springer-Verlag, New York, 1983.

[18] P.H. RABINOWITZ, Existence and nonuniqueness of rectangular solutions of the Bénard problem, Arch. Rational Mech. Anal., 29 (1968), pp. 32-57.

[19] M. SAlBy, Fundamentals of atmospheric physics, Academic Press, 1996.

[20] S. ScheEl AND N. SEeHAFER, Bifurcation to oscillations in three-dimensional Rayleigh-Bénard convection, Phys. Rev. E, vol.56, No.5 (1997), pp. 5511-5516.

[21] M. Schnaubelt And F. Busse, On the stability of two-dimensional convection rolls in an infinite Prandtl number fluid with stress-free boundaries, J. Appl. Math. Phys., vol.40, (1989), pp. 153-162.

[22] R. TEMAM, Infinite-dimensional dynamical systems in mechanics and physics, vo. 68 of Applied Mathematical Sciences, Springer Verlag, New York, second ed., 1997.

[23] X. Wang, Infinite Prandtl number limit of Rayleigh-Bénard convection, Comm. Pure Appl. Math., vol.57, (2004), pp. 1265-1282.

[24] - A note on long time behavior of solutions to the Boussinesq system at large Prandtl number, Contemporary Mathematics (2005), pp. 315-323.

[25] T. Yanagisawa And Y. Yamagishi, Rayleigh-Bénard convection in spherical shell with infinite Prandtl number at high Rayleigh number, J. Earth Simulator, vol.4, (2005), pp. 11-17.

Department of Mathematics, New York Institute of Technology, Old WestBURY, NY 11568

E-mail address: jpark10@nyit.edu 